\title{
A Neural Network Method for Detection of Obstructive Sleep Apnea and Narcolepsy Based on Pupil Size and EEG
}

\author{
Derong Liu, Fellow, IEEE, Zhongyu Pang, Student Member, IEEE, and Stephen R. Lloyd
}

\begin{abstract}
Electroencephalogram (EEG) is able to indicate states of mental activity ranging from concentrated cognitive efforts to sleepiness. Such mental activity can be reflected by EEG energy. In particular, intrusion of EEG theta wave activity into the beta activity of active wakefulness has been interpreted as ensuing sleepiness. Pupil behavior can also provide information regarding alertness. This paper develops an innovative signal classification method that is capable of differentiating subjects with sleep disorders which cause excessive daytime sleepiness (EDS) from normal control subjects who do not have a sleep disorder based on EEG and pupil size. Subjects with sleep disorders include persons with untreated obstructive sleep apnea (OSA) and narcolepsy. The Yoss pupil staging rule is used to scale levels of wakefulness and at the same time theta energy ratios are calculated from the same 2-s sliding windows by Fourier or wavelet transforms. Then, an artificial neural network (NN) of modified adaptive resonance theory (ART2) is utilized to identify the two groups within a combined group of subjects including those with OSA and healthy controls. This grouping from the $\mathrm{NN}$ is then compared with the actual diagnostic classification of subjects as OSA or controls and is found to be $91 \%$ accurate in differentiating between the two groups. The same algorithm results in $90 \%$ correct differentiation between narcoleptic and control subjects.
\end{abstract}

Index Terms-Adaptive resonance theory (ART2), electroencephalogram (EEG), healthy controls, narcolepsy, neural network (NN), obstructive sleep apnea (OSA), pupil stage.

\section{INTRODUCTION}

$\mathbf{S}$ LEEP plays an important role in the history of neuroscience and in the lives of human beings. Excessive daytime sleepiness (EDS) caused by two major sleep disorders, sleep apnea and narcolepsy, can have a disruptive, embarrassing, or even dangerous impact on daily living activities. Sleep apnea is a sleep-related breathing disorder which disrupts normal sleep. Narcolepsy is a neurological disorder which causes irresistible urges to sleep during normal waking hours as well as disruptions in nighttime sleep [29]. Specifically, obstructive sleep apnea (OSA), the most common type of sleep apnea, is a clinical syndrome characterized by recurring episodes of upper airway nar-

Manuscript received May 13, 2006; revised May 2, 2007 and June 24, 2007; accepted July 2, 2007.

D. Liu and Z. Pang are with the Department of Electrical and Computer Engineering, University of Illinois at Chicago, Chicago, IL 60607-7053 USA (e-mail: dliu@ece.uic.edu; zpang@ @il.ece.uic.edu).

S. R. Lloyd is with the Center for Narcolepsy, Sleep, and Health Research, College of Nursing, University of Illinois at Chicago, Chicago, IL 60612-7350 USA (e-mail: srlloyd@uic.edu).

Color versions of one or more of the figures in this paper are available online at http://ieeexplore.ieee.org.

Digital Object Identifier 10.1109/TNN.2007.908634 rowing and/or closure during sleep. Sleep apnea is a serious public health problem. The National Heart, Lung, and Blood Institute (NHLBI, Bethesda, MD) estimates the prevalence rate of sleep apnea at approximately $6.62 \%$ or 18 million Americans at any given time. In the United States and Europe, approximately $75 \%$ of people seen in sleep disorder clinics have OSA [35]. OSA is associated with a high degree of morbidity, including growth retardation (in children), neurobehavioral deficiencies, and cardiovascular complications [37].

Symptoms of OSA include loud and irregular snoring, restless sleep, and daytime sleepiness. In addition, episodes of sleep apnea are often associated with oxygen desaturation. Repeated episodes of this desaturation can eventually lead to additional medical complications. Sleep apnea is clinically diagnosed when those symptoms are present and an all-night sleep study reveals the presence of at least five episodes of apnea and/or hypopnea per hour of sleep.

Narcolepsy is a neurologic disorder in which people experience instability in the sleep-wake cycle. It is characterized by problematic sleepiness during waking hours and disturbed nighttime sleep. Narcolepsy is a serious clinical problem that usually begins before age 25 and persists throughout the lifetime. The estimated prevalence is about one person per 1359 or $0.07 \%$ of the population, with men and women equally affected [23], [43]. About 20\% of persons seen in sleep disorder clinics in the United States and Europe have narcolepsy [35]. Sleep attacks, brief episodes (often 15 min or less) of sleep that occur at any time of the day, and certain manifestations of the auxiliary symptoms of narcolepsy appear to be closely related to the neurophysiologic mechanisms of rapid eye movement (REM) sleep. Sleep attacks may involve a dysfunction of the activating part of the reticular formation in addition to REM mechanisms [3]. As a result of their illness, narcoleptic patients often show symptoms of psychopathology [22]. At the present time, the multiple sleep latency test (MSLT) is the polygraphic EEG (electroencphalogram) "gold standard" for objectively measuring physiologic sleepiness. The standard diagnostic procedure for narcolepsy is time consuming and complex: a 14-day, medication-free period followed by a 7-day period of normal sleep-wake cycles. Then, an all night polysomnographic sleep study is followed by a day-long MSLT [1], [7]. Thus, narcolepsy can be diagnosed when 1) an all-night sleep study (polysomnography) is negative for the presence of another sleep disorder and 2) MSLT nap study findings include a mean sleep latency of less than $5 \mathrm{~min}$ and in at least two of the nap periods REM sleep must occur within $15 \mathrm{~min}$ of patient falling asleep [2]; so an alternative, reliable, easily administered, and simple test is clearly desirable. The social and economic burdens for undiagnosed or misdiag- 
nosed narcolepsy is more than enough to justify the research aimed at finding such tests [33].

In general, there are two classes of detection methods that have been applied to diagnostic methods for OSA. One class is based on breath signals while the other is based on electromyogram (EMG) signals. Several research papers [13], [14], [38], [42], [45] have examined methods belonging to the first class. Assessment of respiratory signal is a key step to diagnose OSA. Respiratory impedance can be determined by a forced oscillation technique (FOT) [14] and can be considered as a proper noninvasive method to diagnose sleep apnea. FOT relies on applying an oscillatory pressure signal to the respiratory system and determines the respiratory impedance using nasal pressure and airflow signals. Currently, FOT is a promising noninvasive method for measuring respiratory impedance [13], [38]. Using FOT during sleep, Yen et al. [45] estimated airway impedance with high specificity and reliability. Then, they used an artificial neural network (NN) to classify people with and without hypopnea/apnea based on this respiratory signal. Gumery et al. [19] developed a device to measure the surface EMG time latency reflex of the genioglossus muscle stimulated by time and amplitude calibrated with negative pharyngeal pressure drops. Then, based on a Berkner transform, they built a multiscale detector. Further, they tested those detectors in terms of accuracy and robustness using signals acquired from apneic patients and healthy controls.

Zorick [48] investigated some properties of 103 patients with narcolepsy including intrusion of sleep into awake time. Results showed that patients with narcolepsy had frequent sleep onset REM periods, $70 \%$ on the MSLT and $45 \%$ on nocturnal recordings. Since EEG and pupil diameter can represent this change, they indicate that the EEG and pupil diameters of narcoleptic patients differ significantly from normal at the onset of sleep. Another possible basis for a narcolepsy test is to recognize the relationship between sleepiness and pupil diameter which indicates that pupil size decreases with advancing sleepiness [46].

EEG measurement can be used to detect brain activity since different mental activities produce different EEG patterns. Millán et al. [31] present a neural classifier to recognize mental tasks and get about $70 \%$ correct recognition. Researchers have found that fluctuations in wakefulness can be examined with EEG measurement from active subjects with eyes open and engaged in their usual awake activities [4], [20]. In these situations, intrusions of alpha and theta activity into the beta activity of active wakefulness have been interpreted as ensuing sleepiness. As a further characterization of a sleepy EEG pattern, Guilleminault [18] defined lapses into microsleeps with ensuing sleepiness as the appearance of 3-14-s bursts of theta activity into the awake EEG. A microsleep [18] is defined as the transient period of decreased wakefulness that can occur prior to sleep onset and is often found in sleepy individuals. Keegan and Merritt [24] found a significant correlation between variations in pupil size and EEG power measures for the delta, theta, alpha, and beta bands, respectively, when data were segmented into 2-s windows. Yoss et al. [46] developed a system for scaling levels of wakefulness during $15 \mathrm{~min}$ of alertness testing by measuring pupil diameter as a proportion of the individual's maximum pupil diameter (stage 1 "awake" $=95 \%-100 \%$, stage $2=85 \%-94 \%$, stage $3=75 \%-84 \%$, and stage 4 "sleepy" $=65 \%-74 \%$ of maximum diameter).
Subjects with OSA, narcolepsy, and healthy controls may have different alertness levels under the same conditions. The pupil response patterns between subjects with and without sleep disorders are different [27]. In this paper, we develop a novel method to detect subjects with sleep disorders based on EEG and pupil size. We compare subjects with sleep disorders to those healthy controls and find that they have different responses in theta wave patterns for the same situation. A significant difference between those subjects can be used for the purpose of diagnosis by artificial NNs, specifically, modified adaptive resonance theory (ART2) NNs. Compared with simulation results of backpropagation NNs and Hopfield NNs, modified ART2 NNs give us much better results for this project. We tested our algorithm using two sets of subjects including one set of five subjects with OSA plus six healthy controls and another set of four subjects with narcolepsy plus six healthy controls. This methodology may eventually lead to new diagnostic methods for specific sleep disorders such as OSA, narcolepsy, etc. To the best of our knowledge, no researchers have reported the classification of patients with OSA or with narcolepsy by NN methods based on EEG and pupil size data.

This paper is organized as follows. In Section II, we present the experimental data. In Section III, data preprocessing is described and our method for detecting excessive daytime sleepiness associated with sleep disorders is developed. In Section IV, simulation results are given. In Section V, conclusions are presented and future perspectives are discussed.

\section{EXPERIMENTAL DATA COLLECTION}

Data from five untreated OSA subjects, four narcoleptic subjects, and six healthy controls were collected approximately $12 \mathrm{~h}$ after their midsleep period to maximize the probability of sleepiness occurring. This midafternoon increase in somnolence, commonly believed to be a postprandial phenomenon, has been shown to be unrelated to food intake [6]. Data collection was performed at the Center for Narcolepsy Research, University of Illinois at Chicago, Chicago, IL. The alertness level testing, conducted with a pupillometry system built at Mayo Clinic [28], consists of 1-min recording of pupil diameter in the light followed by about $14 \mathrm{~min}$ in a quiet, dark room. During the alertness level testing, subjects were seated in a comfortable chair and instructed to stay awake but not to do anything special to maintain alertness, to try to minimize blinking, and to stare straight ahead at a small red dot projected on a wall approximately $2 \mathrm{~m}$ from their eyes. The video pupil images from two small infrared cameras are processed by an image processor which brightened the outline of the pupil circumference. The image processor detects and outputs a voltage proportional to the pupil diameter. The analog pupil diameter data are digitized at the rate of $256 \mathrm{~Hz}$ using an analog-to-digital (A/D) converter and saved to a personal computer (PC) using a binary format [28]. EEG/polysomnography (PSG) data were recorded simultaneously with the pupil diameter data using a Grass Instruments (Quincy, MA) model 8-16D polygraph and the standard MSLT montage: EEG (C3/A2, O1/A2, and $\mathrm{P} 3 / \mathrm{O} 1$ ), electro-oculogram (EOG) [right oculogram (ROC/A1) and left oculogram (LOC/A2)] and EMG (bilateral mentalis placement) [8]. Filters for EEG and EOG were set at $0.3 \mathrm{~Hz}$ for high pass and $30 \mathrm{~Hz}$ for low pass. EMG filters were set at 10 


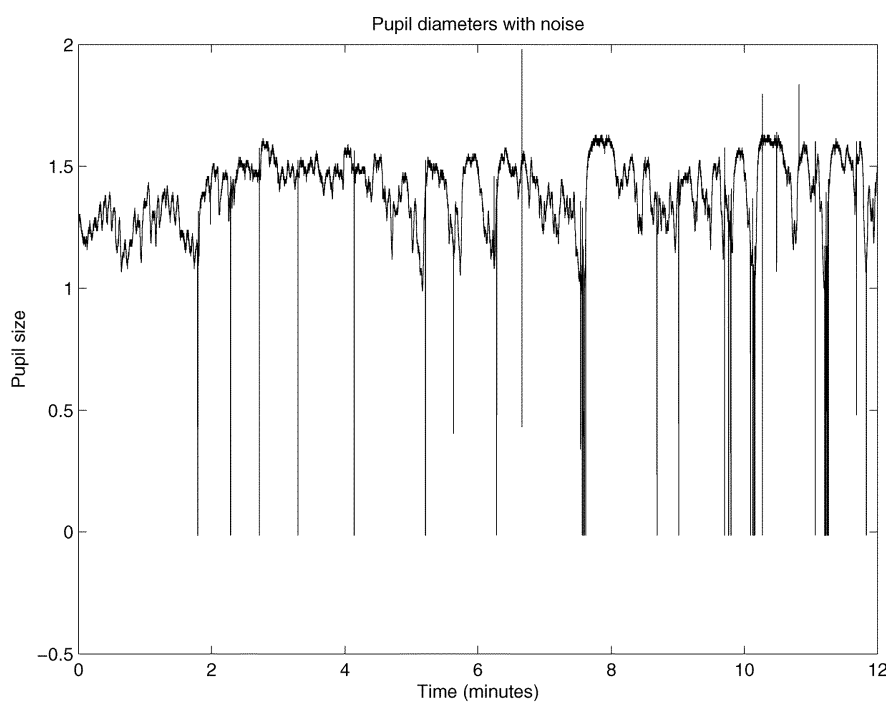

(a)

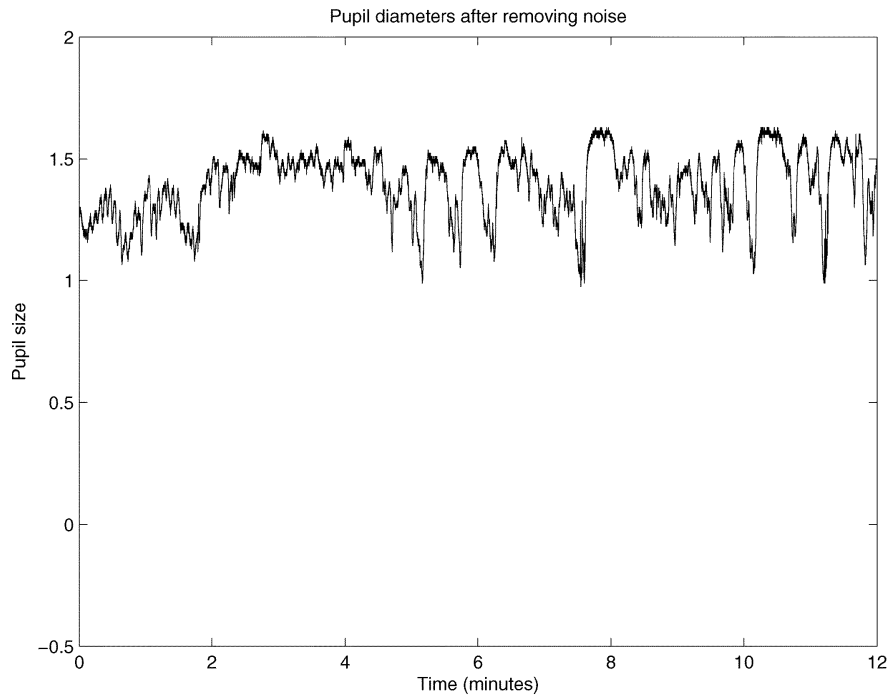

(b)

Fig. 1. Comparison between data of pupil size (a) before and (b) after noise removal.

and $100 \mathrm{~Hz}$, respectively. EEG/PSG data were also digitized at $256 \mathrm{~Hz}$ and stored with pupillometry data in a PC.

\section{METHOD FOR DETECTING SLEEP DisORDER BASED ON ART2 NNS}

\section{A. Data Preprocessing}

Pupil size data contains "noise" such as blinking and eye movements since they cannot be avoided during data recording. These artifacts are not useful for our method and do not contain any useful information. We designed an algorithm to remove these artifacts and used linear interpolation to place data in the resulting time gaps in order to get a continuous time series. In particular, when pupil diameters reach zero or pupil diameters in two adjacent time points change too much, we consider them as abnormal data. Fig. 1 shows an example of pupil diameter data of a subject before and after removing the artifacts. Our method is similar to that developed by Merritt et al. [30].
In accordance with Yoss pupil staging method, we used a sliding window of $2 \mathrm{~s}$. Data recording from each subject has $14.5 \mathrm{~min}$ and the first $3 \mathrm{~min}$ are ignored. Thus, there are 11.5 min of data for analysis. Data for the first 3 min of recording were eliminated from analysis because the pupil dilates and oscillates when the lights are extinguished and it takes 2-3 min of darkness to adapt and reach a larger stable diameter [25]. The largest pupil diameter is normally found during the fourth minute of dark recording. Thus, the mean pupil diameter of each 2-s window can be calculated and divided by the largest pupil diameter to get pupil size ratios for Yoss stages.

On the other hand, theta wave activity $(4-8 \mathrm{~Hz})$ is an indicator of sleepiness. The amount of theta wave activity has been shown to increase in value during episodes where people demonstrate decreasing alertness level. Accordingly, theta energy was calculated for 2-s windows with the original data from three EEG channels of C3/A2, O1/A2, and P3/O1. We use both Fourier and wavelet transforms on each data set and find that Fourier transform has good performance for OSA while wavelet transform gives better results for narcolepsy. Therefore, for subjects with OSA and accompanying controls, Fourier transform is used to get theta wave and further to obtain theta energy. For subjects with narcolepsy and accompanying controls, wavelet transform is applied to get theta wave and further to obtain theta energy. We used Matlab to realize these procedure. After that, the average energy can be obtained for each sliding window, which indicates the amount of theta wave present. Theta energy data are divided by the mean power value of fourth minute to obtain theta energy ratios, because pupil size is normally the biggest in the fourth minute. In order to find the information of sleepiness with changing pupil size, we realign power ratio data of theta waves according to pupil stages. Since some unknown artifacts may exist in the EEG data, eliminating all artifacts was not possible. Thus, we use the regression method to catch changes of theta activity following pupil stages. The regression analysis is based on the method of Chatterjee and Hadi [9], expressed by

$$
Y=X \beta+\epsilon, \quad \epsilon \sim N\left(0, \sigma^{2} I\right)
$$

where $Y$ is a dependent variable (output), $X$ is an independent variable (input or data), and $\epsilon$ is the error. Solving for $\beta$ from (1) based on the least square error will give the predicted data.

\section{B. ART2 NNS}

ART was introduced by Grossberg [17] in 1976. ART2 NNs [5] were designed for both analog and digital inputs in 1987. ART2 has been widely used to identify patterns in various fields, e.g., Suzuki [40] used NNs based on ART2 to recognize QRS waves $^{1}$ from electrocardiogram (ECG); Vlajic and Card [44] make use of a modified ART2 NNs to discover input data clusters without considering their actual size; and Chen et al. [10] incorporate the incremental communication method into ART2 NNs to reduce the communication cost. This paper is based on ART2 NNs with modified learning functions to adapt to the input patterns. Fig. 2 shows the architecture of ART2. An ART2 NN [5], [12] consists of two subsystems: an attentional subsystem and an orienting subsystem. The attentional subsystem

\footnotetext{
${ }^{1} \mathrm{QRS}$ wave is a peak pattern in ECG which is made up by three points of $\mathrm{Q}$, $R$, and $S$, where $Q$ is the left bottom point of the wave, $R$ is the peak point of the wave, and $\mathrm{S}$ is the right bottom point of the wave.
} 


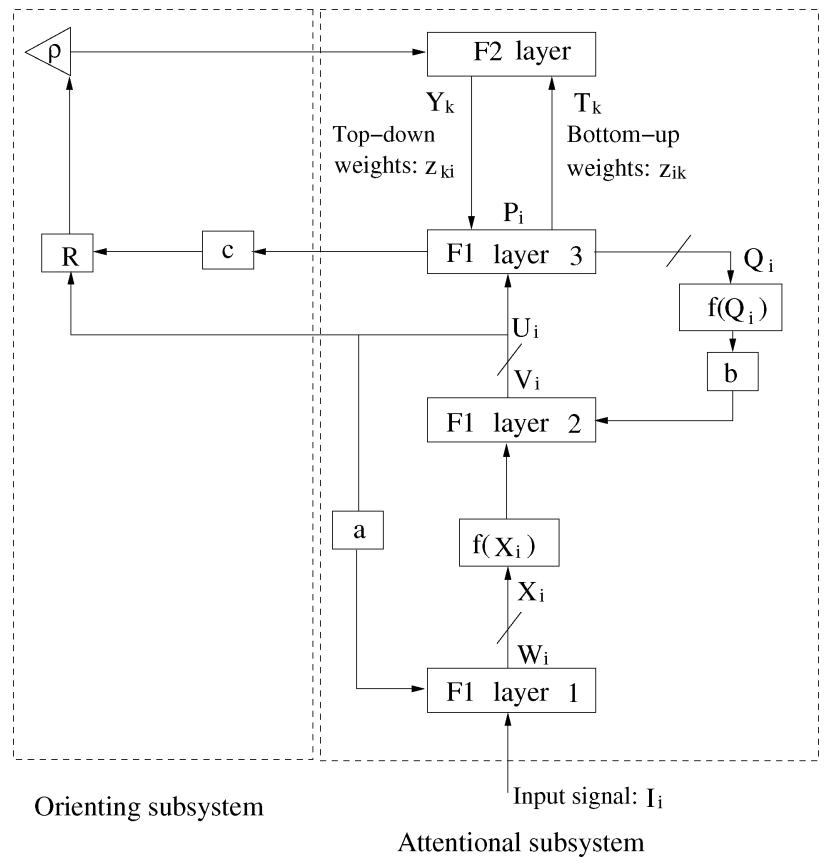

Fig. 2. ART2 NNs architecture.

has two layers, F1 and F2. F1 is made up of three sublayers. Here, three sublayers of F1 are necessary for analog input patterns since the differences between possible signals with particular patterns may be much smaller for analog inputs than for binary inputs which are used to represent features of signals. The signals of Fig. 2 are realized individually by some equations; see [5] for details.

One function in category layer (F2) is given as

$$
T_{k}=\sum_{i} p_{i} z_{i k}
$$

where $T_{k}$ is the value of the $k$ th category layer from the third layer, $z_{i k}$ is the weight from the $i$ th input of the third layer of $\mathrm{F} 1$ to the $k$ th category in the category layer, and $p_{n}$ is the signal from the third layer of $\mathrm{F} 1$. The contrast-enhancing function $f_{\mathrm{cn}}$ is a choice function and can use sigmoid function. In our case, the contrast-enhancing function is defined by

$$
f_{\text {cn }}\left(y_{j i}\right)= \begin{cases}d, & \text { if } t_{j}=\max _{k}\left\{t_{k i}\right\} \\ 0, & \text { otherwise }\end{cases}
$$

where $d$ is a parameter to be determined and $t_{k i}$ is defined in (2). When resonant conditions exiting in the network are below the threshold set by the vigilance parameter, the memory will be activated and the long-term memory (top-down weights and bottom-up weights) adaptive process described in the following will also be activated.

The following architecture describes the updating relationship between the third layer of F1 from an input signal and the activated category layer.

Bottom-up long-term memory trace $(\mathrm{F} 1 \rightarrow \mathrm{F} 2)$

$$
\frac{d}{d t} z_{i j}=f_{\mathrm{cn}}\left(y_{j}\right)\left[p_{i}-z_{i j}\right] .
$$

Top-down long-term memory trace $(\mathrm{F} 2 \rightarrow \mathrm{F} 1)$

$$
\frac{d}{d t} z_{j i}=f_{\mathrm{cn}}\left(y_{j}\right)\left[p_{i}-z_{j i}\right]
$$

where $p_{i}$ is the $i$ th output of the third layer of $\mathrm{F} 1, y_{j}$ is the output of the $j$ th category activated, and $z_{j i}$ and $z_{i j}$ are the weights between the category of the layer F2 and the third layer of F1.

When resonant conditions existing in the network exceed the threshold set by the vigilance parameter, we modify the original update equations in order to avoid forgetting all the information obtained before. The memory will be updated by the average value of all long-term memory (LTM) associated with the same winner while individual input should get its own LTM by (6) and (7). The memory update is described as follows. Bottom-up long-term memory trace $(\mathrm{F} 1 \rightarrow \mathrm{F} 2)$

$$
z_{i j} \Leftarrow \frac{n-1}{n} z_{i j}+\frac{1}{n} z_{i j}^{\prime} .
$$

Top-down long-term memory trace $(\mathrm{F} 2 \rightarrow \mathrm{F} 1)$

$$
z_{j i} \Leftarrow \frac{n-1}{n} z_{j i}+\frac{1}{n} z_{j i}^{\prime} .
$$

In (6) and (7), $n$ is the number of subjects associated with the winner $j, z_{i j}$ in $((n-1) / n) z_{i j}$ and $z_{j i}$ in $((n-1) / n) z_{j i}$, respectively, are the weight before update, and $z_{i j}^{\prime}$ and $z_{j i}^{\prime}$, respectively, are the new weight from a new input.

Reset equations in the orienting subsystem are the key part of ART2 NN since it is related to the ability of identification. We use the same equations as Grossberg's ART2 [5], given by

$$
\begin{aligned}
r_{k} & =\frac{u_{k}+c p_{k}}{e+\left\|U_{i}\right\|+c\left\|P_{i}\right\|} \\
R_{i} & =R\left(U_{i}, P_{i}\right), \\
\left\|R_{i}\right\|+e & <\rho
\end{aligned}
$$

where $\rho$ is a vigilance parameter, $r_{k}$ is the $k$ th element of similarity vector $R_{i}, e$ is a small number, $u_{k}$ and $p_{k}$ are the $k$ th element of $U_{i}$ and $P_{i}$, respectively, and $U_{i}$ and $P_{i}$ are signals in Fig. 2.

\section{Our System and Parameter Selection}

Since the vigilance parameter $\rho$ decides the level of similarity between input signals in the same category, more categories will be obtained if $\rho$ is large when other parameters are the same, e.g., if $\rho$ is close to 1 . The order of input signals has certain effects on the final classification results. For example, three input signals $\mathrm{A}, \mathrm{B}$, and $\mathrm{C}$ are given and $\mathrm{A}$ and $\mathrm{B}$ are very similar while $\mathrm{B}$ and $\mathrm{C}$ are very similar, but $\mathrm{A}$ is far away from $\mathrm{C}$ compared to between $\mathrm{B}$ and $\mathrm{C}$. Under certain model parameters, if $\mathrm{A}$ is the first input to ART2, B and C will be grouped together with A. However, if $\mathrm{B}$ is the first input signal, $\mathrm{A}$ is the second, and $\mathrm{C}$ is the third, A and B may be grouped together while $\mathrm{C}$ is in a different group. The reason is that the original ART2 algorithms has forgetting property. In order to solve this problem, a large $\rho$ is chosen so that only signals similar enough will be grouped together. Based on (6) and (7), a mean signal can be obtained in one group. After that, the other ART2 are used with a different set of parameters to classify grouped signals from the first ART2. The parameter choices for our method are based on the original ART2 paper [5], where their relationship is derived and limits are set. Based on their relationship and limits, we use the 


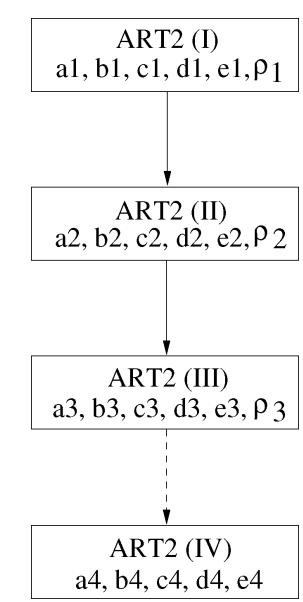

Fig. 3. Architecture of four ART2 series. Dashed line arrowhead represents that the fourth one exits if it is necessary.

method of trial and error and choose a set of best parameters for our system.

Selection of parameters $c$ and $d$ in the system must obey certain constraints according to [5], where a proof was given for constraint given by

$$
\frac{c d}{1-d} \leq 1
$$

We choose different values of $c$ and $d$ for different input types. The parameters $a$ and $b$ in Fig. 2 may have a more significant impact on the stability of the network. A few papers [5], [12] discussed the effect of parameters $a$ and $b$. In general, small $a$ and $b$ could make the network unstable and the values of parameters $a$ and $b$ affect the range of the vigilance parameter $\rho$. The smaller the values of $a$ and $b$ are, the wider the usable range of the vigilance parameter is. If values of $a$ and $b$ are too big, sensitivity of the network will decrease significantly, and the number of members in a group will increase. The vigilance parameter has a critical effect on the results of classification. A bigger value of the vigilance parameter tends to separate inputs into more categories. Only very similar subjects can be grouped together. On the other hand, if its value is too small, most inputs will go into one group; so we use three ART2 networks in hierarchy for subjects with OSA or narcoleptic subjects, respectively, in order to avoid missing some subjects due to the choice of vigilance parameter and to obtain more precise classifications. After that, if there are still more than two groups, a fourth ART2 may be necessary as in Fig. 3. We tried to use the smallest number of layers possible for each data set to classify them successfully. After resulting comparison of different number of layers of our system, we found that three layers for OSA data had good performance and so did four layers for narcoleptic data. In each ART2, we use the same structure as in [5] shown in Fig. 2. However, we do not follow the procedure of traditional ART2 and we separate these subjects into two groups based on their similarity parameters for the fact that the larger the similarity parameter is, the closer they are to each other.

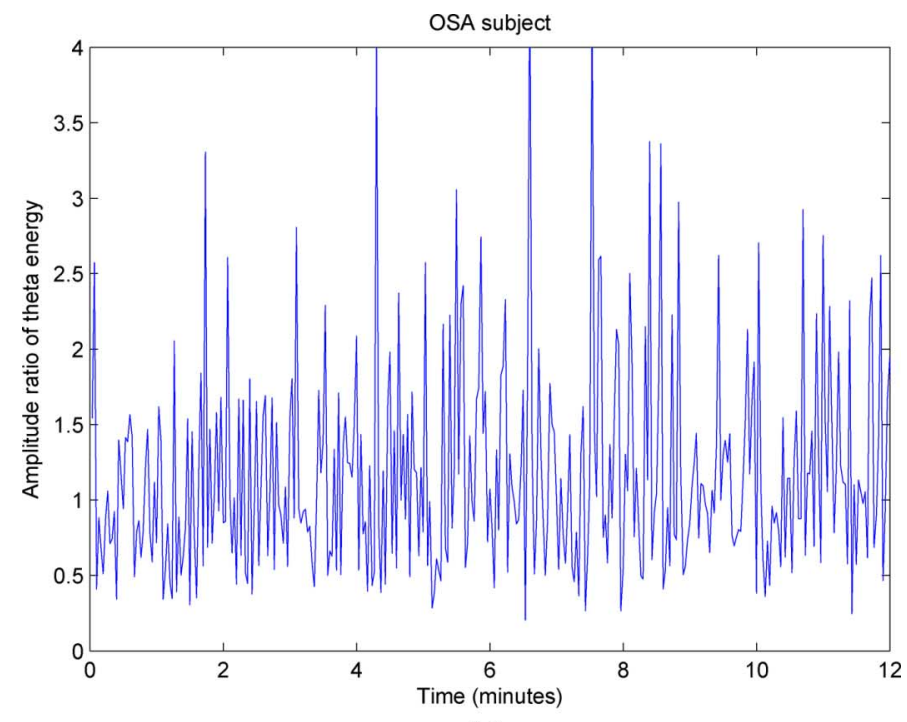

(a)

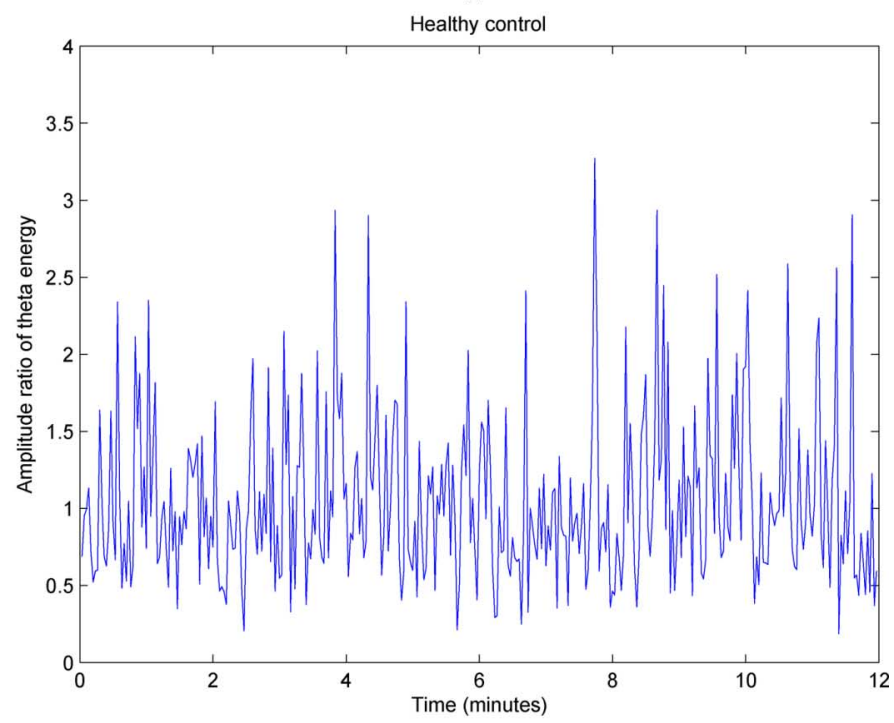

(b)

Fig. 4. Comparison of theta energy ratio between (a) a subject with OSA and (b) healthy control.

\section{Simulation RESUlts}

\section{A. Classification Between Subjects With OSA and Controls}

A total of 11 subjects are used to test our NN algorithm, including six healthy controls and five subjects with OSA. Ten of the 11 subjects are correctly classified so the differentiation rate is $91 \%$. Fig. 4 shows the energy ratio distribution of the theta wave of one subject with OSA and one healthy subject after aligning data according to pupil stages.

It is not possible to distinguish them from the figures, so we use linear regression method to process them further. Fig. 5 shows the results of regression with different point numbers for a healthy control and an OSA subject. Fig. 6 shows the pupil stages of the same subject.

The patterns have discrepancy between different subjects. The energy ratio of theta wave is from the original data with 2-s sliding windows, in which artifacts such as eye blinking have 


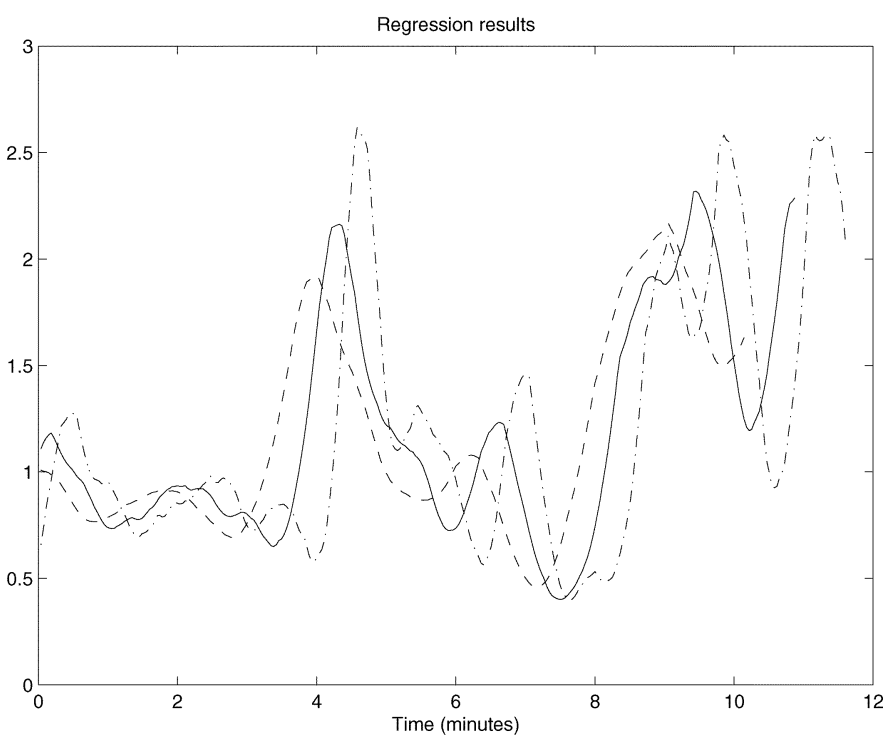

(a)

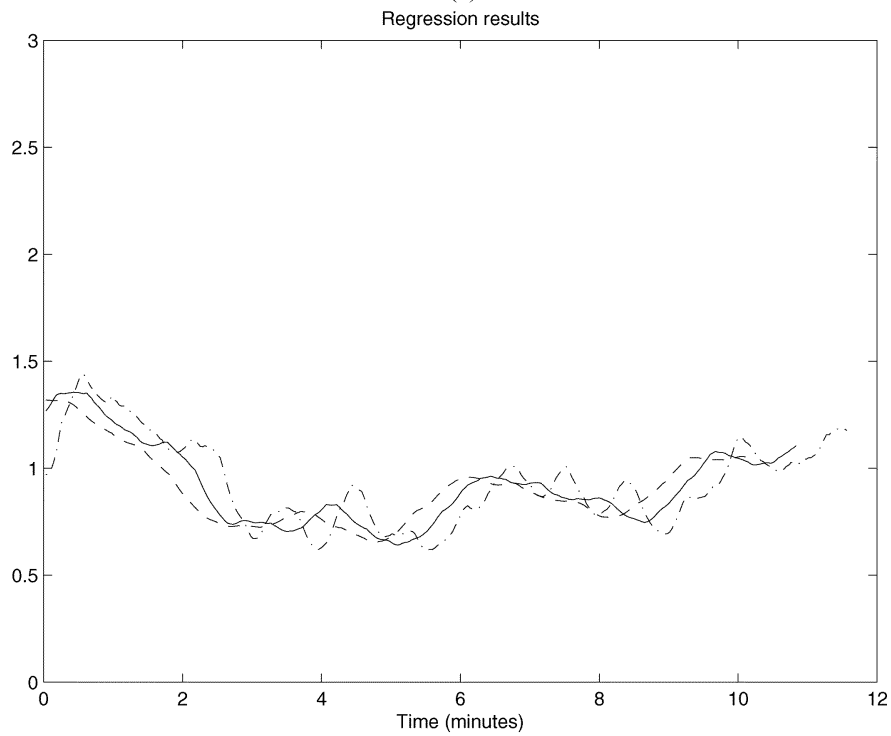

(b)

Fig. 5. Regression results of an OSA subject and a healthy control with 20 , 30 , and 40 regression points. (a) OSA subject and (b) healthy control. Dash-dot lines represent results with 20 regression points, solid lines are for 30 regression points, and dashed lines show results for 40 regression points.

been removed. However, the regression procedure can make the change of theta energy ratio more obvious.

One ART2 NN with another ART2 cannot identify all subjects and more subjects go to the wrong group since there are subjects grouped into more categories or missed as a noise under a fixed vigilance parameter $\rho$. If parameter $\rho$ is set slightly higher, more subjects will not be classified. On the other hand, if the parameter $\rho$ is set slightly lower, most subjects go into the same group. That is, the accuracy of classification will be reduced. Tables I and II show parameters we used in traditional ART2 NN and three ART2 series. In addition, we find that regression by different data points has an effect on the results. The bigger the number of points used in the regression is, the higher the vigilance parameter $\rho$ has to be set in order to get better classification results. More points make the figure more flat and stable. For purpose of comparison of one ART2 and

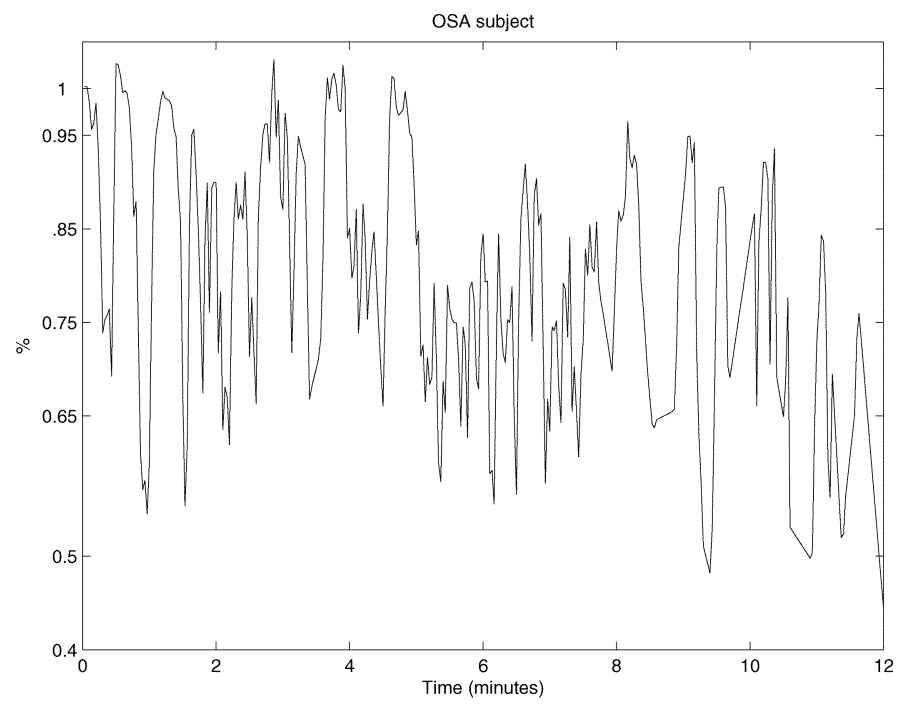

(a)

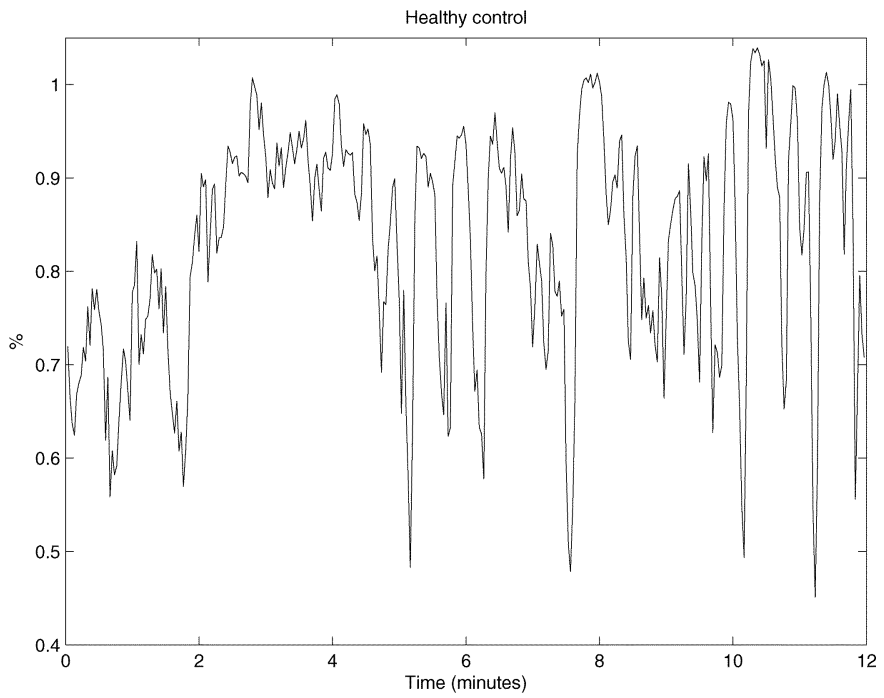

(b)

Fig. 6. Comparison of pupil stages between a subject with (a) OSA and (b) healthy control.

TABLE I

Parameters of ONE TRAditional ART2

\begin{tabular}{|c|c|c|c|c|c|c|}
\hline $\begin{array}{c}\text { No. of } \\
\text { points }\end{array}$ & 10 & 20 & 25 & 30 & 35 & 40 \\
\hline $\mathrm{a}$ & 2 & 2 & 2 & 2 & 2 & 2 \\
\hline $\mathrm{b}$ & 10 & 10 & 10 & 10 & 10 & 10 \\
\hline $\mathrm{c}$ & 0.1 & 0.1 & 0.1 & 0.1 & 0.1 & 0.1 \\
\hline $\mathrm{d}$ & 0.9 & 0.9 & 0.9 & 0.9 & 0.9 & 0.9 \\
\hline$\rho$ & 0.88 & 0.84 & 0.835 & 0.83 & 0.825 & 0.82 \\
\hline $\mathrm{e}$ & 0.001 & 0.001 & 0.001 & 0.001 & 0.001 & 0.001 \\
\hline
\end{tabular}

Each point represents $2 \mathrm{~s}$. ART2 parameters do not have any units.

three ART2 series, we draw the figure of performance in Fig. 7. The vertical axis is the percentage of successful classification while the horizontal axis is the number of points used in the regression.

From Fig. 7, we find that the three ART2 series could get much better result than only one ART2 under large vigilance parameter $\rho$. The reason is from the architecture of ART2 which reflects the similarity of signals. A single vigilance parameter $\rho$ 
TABLE II

PARAMETERS OF ART2 SERIES

\begin{tabular}{|c|c|c|c|c|c|c|}
\hline \multirow{2}{*}{$\begin{array}{c}\text { Number of } \\
\text { points }\end{array}$} & \multicolumn{3}{|c|}{10} & \multicolumn{3}{|c|}{20} \\
\hline & S1 & $\mathrm{S} 2$ & S3 & S1 & $\mathrm{S} 2$ & S3 \\
\hline $\mathrm{a}$ & 0.7 & 0.5 & 0.5 & 2 & 2 & 5 \\
\hline $\mathrm{b}$ & 15 & 10 & 10 & 5 & 10 & 10 \\
\hline $\mathrm{c}$ & 0.08 & 0.08 & 0.08 & 0.08 & 0.08 & 0.05 \\
\hline $\mathrm{d}$ & 0.9 & 0.9 & 0.9 & 0.9 & 0.9 & 0.9 \\
\hline$\rho$ & 0.92 & 0.91 & - & 0.93 & 0.925 & - \\
\hline $\mathrm{e}$ & 0.01 & 0.0001 & 0.0001 & 0.01 & 0.0001 & 0.001 \\
\hline \multirow{2}{*}{$\begin{array}{l}\text { Number of } \\
\text { points }\end{array}$} & \multicolumn{3}{|c|}{25} & \multicolumn{3}{|c|}{30} \\
\hline & SI & $\mathrm{S} 2$ & $\mathrm{~S} 3$ & SI & $\mathrm{S} 2$ & $\mathrm{~S} 3$ \\
\hline $\mathrm{a}$ & 10 & 3 & 5 & 10 & 2 & 5 \\
\hline b & 20 & 10 & 10 & 20 & 10 & 10 \\
\hline $\mathrm{c}$ & 0.1 & 0.1 & 0.02 & 0.1 & 0.055 & 0.02 \\
\hline d & 0.9 & 0.9 & 0.9 & 0.9 & 0.9 & 0.9 \\
\hline$\rho$ & 0.965 & 0.929 & - & 0.972 & 0.93 & - \\
\hline $\mathrm{e}$ & 0.01 & 0.0001 & 0.001 & 0.01 & 0.0001 & 0.001 \\
\hline \multirow{2}{*}{$\begin{array}{l}\text { Number of } \\
\text { points }\end{array}$} & \multicolumn{3}{|c|}{35} & \multicolumn{3}{|c|}{40} \\
\hline & SI & S2 & S3 & SI & S2 & S3 \\
\hline $\mathrm{a}$ & 2 & 1 & 5 & 10 & 2 & 5 \\
\hline $\mathrm{b}$ & 15 & 5 & 15 & 20 & 10 & 10 \\
\hline $\mathrm{c}$ & 0.1 & 0.1 & 0.04 & 0.1 & 0.055 & 0.02 \\
\hline d & 0.9 & 0.9 & 0.9 & 0.9 & 0.9 & 0.9 \\
\hline$\rho$ & 0.97 & 0.925 & - & 0.972 & 0.93 & - \\
\hline $\mathrm{e}$ & 0.01 & 0.0001 & 0.001 & 0.01 & 0.0001 & 0.001 \\
\hline
\end{tabular}

Each point represents 2 s. ART2 parameters do not have any units.

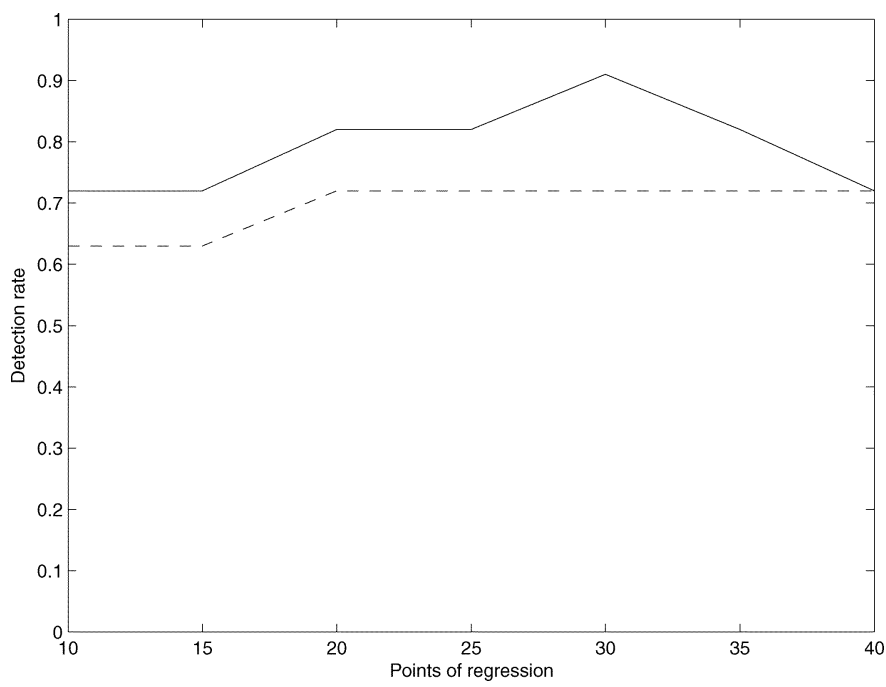

Fig. 7. Performance of one traditional ART2 NN and our system. Solid line represents performance of our system; dashed line represents performance of one traditional ART2.

may not be proper for two different inputs. When the number of inputs is large, one fixed vigilance parameter is not appropriate. Three ART2 series allow us to choose different parameters in different stages. The best performance is from inputs with regression of 30 points. In this case, the first ART2 obtains eight categories, the second ART2 gets three categories, and the third ART2 has two categories. The system could identify correctly ten subjects among 11 subjects. It missed one subject with OSA. After we observed its data, we note that it has a different change of pupil diameter from other subjects. Normally, the pupil diameter of subjects becomes smaller with time during data collection, but this subject has a reverse change. Its pupil diameter becomes the largest in the last $2 \mathrm{~min}$. The reason may be from the angle of camera recording or this subject focuses on the wrong place during the first period of recording. This may be the reason why the subject is misclassified.

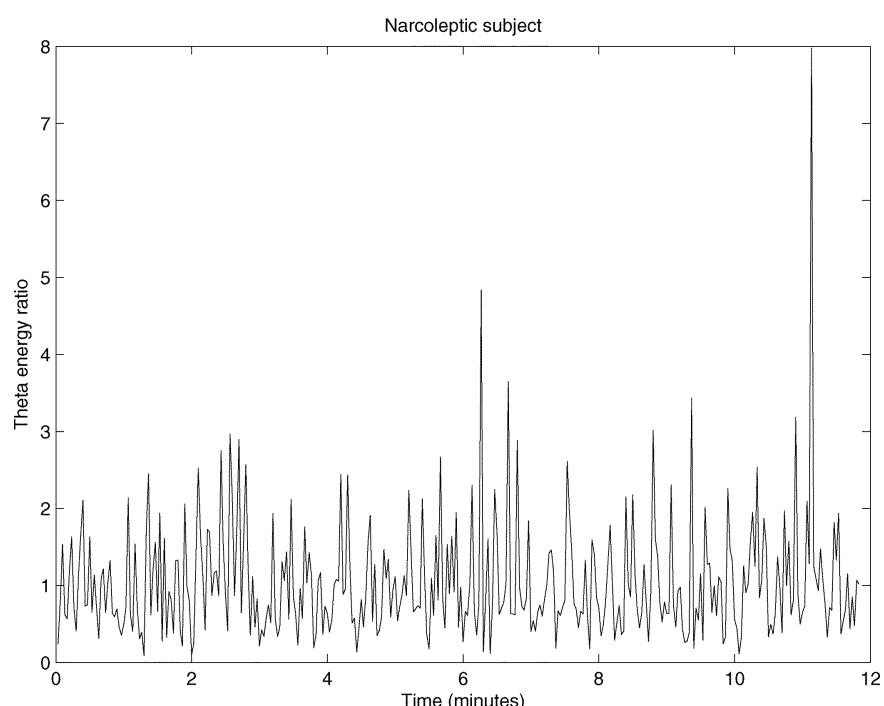

(a)

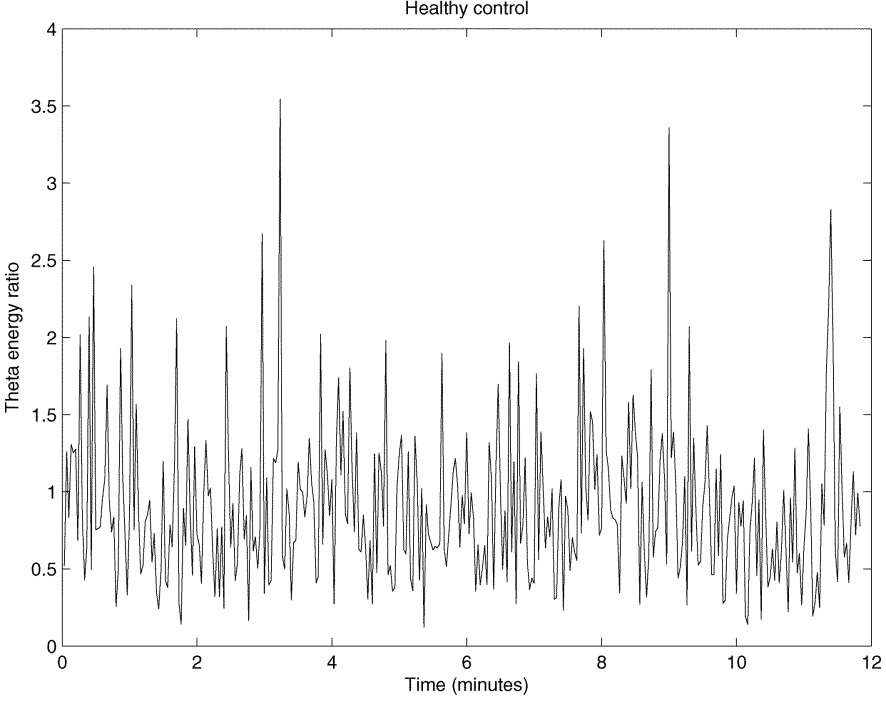

(b)

Fig. 8. Comparison of theta energy ratio between (a) narcoleptic subject and (b) healthy control.

The following example is from our simulation for regression of 30 points. Three ART2 NNs are used and 11 inputs are from 11 subjects. After the first ART2 NNs, 11 inputs go into eight categories since some very similar inputs are grouped together. We average inputs in the same group to get eight inputs for the second ART2. Three categories are obtained after the second ART2, including two big groups and one small group. The same average strategy is applied to these three groups to get three inputs for the third ART2. Finally, two groups are reached after the third ART2. We check the status of each subject in two groups to get the percentage of correct classification.

\section{B. Classification Between Narcoleptic Subjects and Controls}

In order to test our algorithm, we use ten subjects, including six healthy controls and four subjects with narcolepsy. Fig. 8 shows the energy ratio distribution of theta wave of a narcoleptic subject and a healthy subject after aligning data according to pupil stages. 


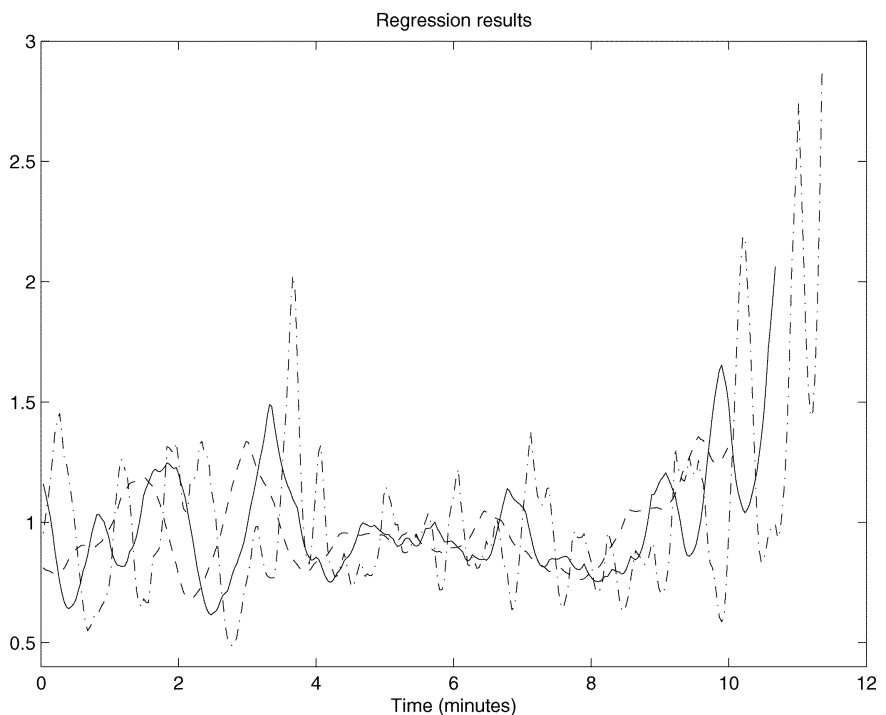

(a)

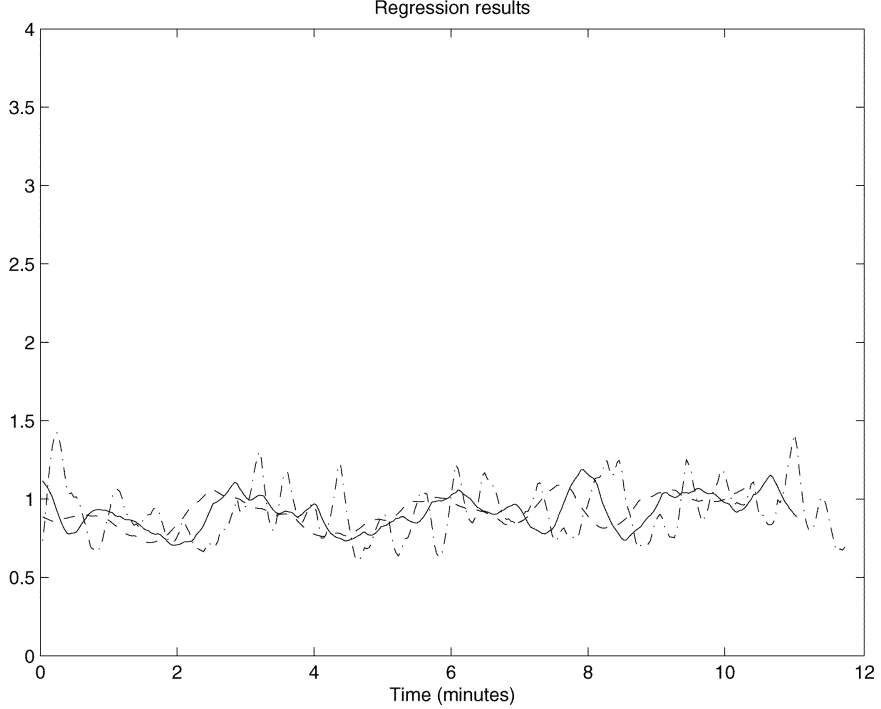

(b)

Fig. 9. Regression results of (a) narcoleptic subject and (b) healthy control with 10,20 , and 30 regression points. Dash-dot lines represent results with ten regression points, solid lines are for 20 regression points, and dashed lines show results for 30 regression points.

Here, the same strategy of the regression method is used to deal with data as in Section IV-A. Fig. 9 shows the results of regressions of different point numbers used for a narcoleptic subject and a healthy control. Fig. 10 shows the pupil stages of a narcoleptic subject and a control. The theta wave is obtained from the original data with 2-s sliding windows by wavelet transform, in which artifacts such as eye blinking are removed before. The energy ratio is further calculated. The regression procedure is used to preprocess data of energy ratio.

The algorithm is similar to the one we used to identify subjects with OSA. The difference is that we use four ART2 series instead of three. We follow the same principle to choose parameters as we did previously to separate subjects with OSA from controls. For purpose of comparison, we first use the traditional ART2 to classify them. Tables III and IV show parameters we used in the traditional ART2 NN and series of four ART2 NNs.

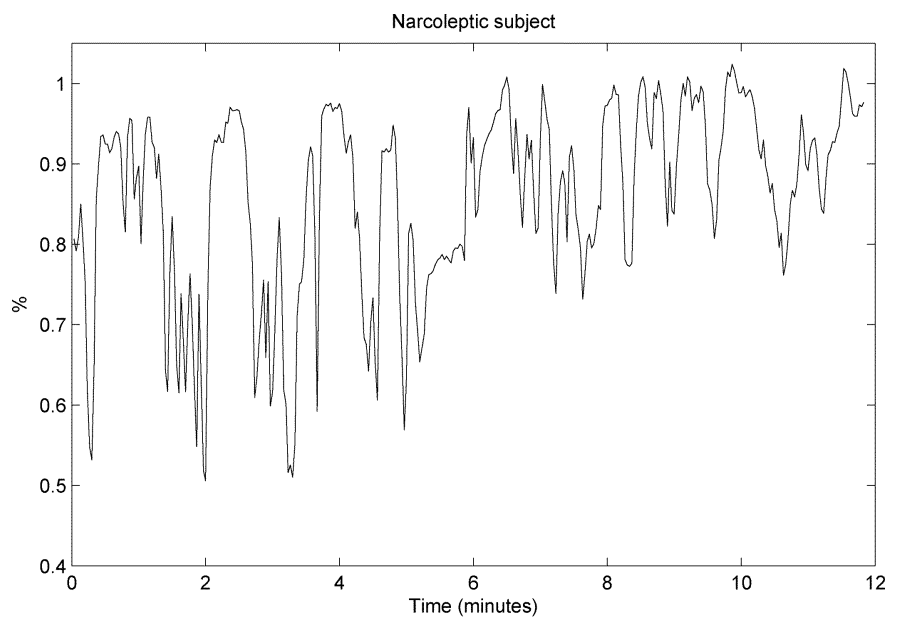

(a)

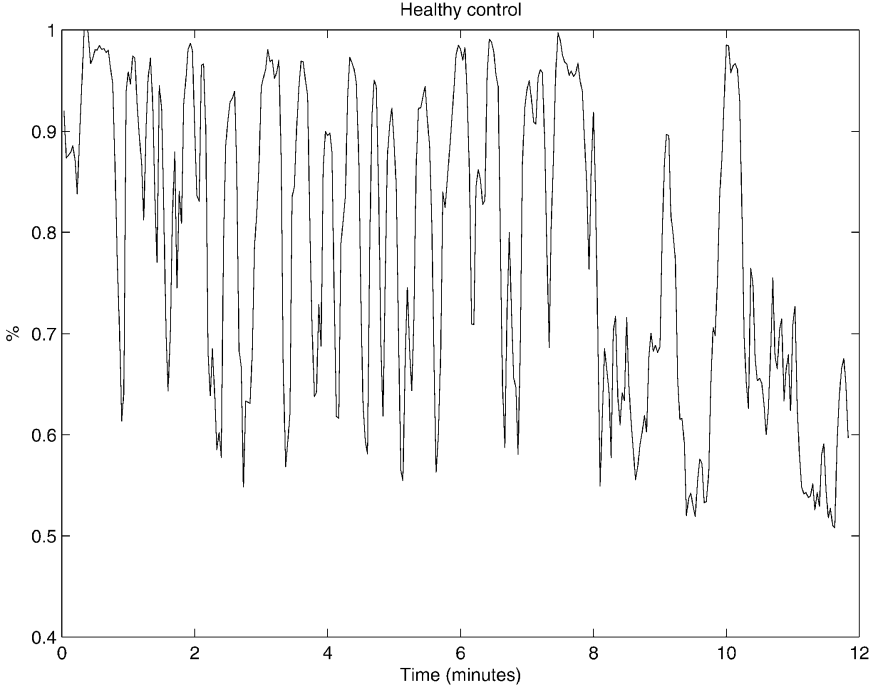

(b)

Fig. 10. Comparison of pupil stages between (a) narcoleptic subject and (b) healthy control.

TABLE III

PARAMETERS OF ONE TRADITIONAL ART2

\begin{tabular}{|c|c|c|c|c|c|}
\hline No. of points & 10 & 20 & 25 & 30 & 40 \\
\hline $\mathrm{a}$ & 10 & 12 & 10 & 8 & 12 \\
\hline $\mathrm{b}$ & 15 & 16 & 13 & 15 & 12 \\
\hline $\mathrm{c}$ & 0.08 & 0.08 & 0.08 & 0.08 & 0.08 \\
\hline $\mathrm{d}$ & 0.9 & 0.9 & 0.9 & 0.9 & 0.9 \\
\hline$\rho$ & 0.895 & 0.905 & 0.92 & 0.915 & 0.9 \\
\hline $\mathrm{e}$ & 0.001 & 0.001 & 0.001 & 0.001 & 0.001 \\
\hline
\end{tabular}

Each point represents $2 \mathrm{~s}$. ART2 parameters do not have any units.

We compare the performance of two methods in Fig. 11. The vertical axis is the percentage of successful classification while the horizontal axis is the number of regression points used.

From Fig. 11, it is obvious that performance of four ART2 series is better than that of only one ART2 under the same inputs. The reason is that flexibility of ART2 series makes it adaptable to this kind of analog input. The best performance is from inputs of 20 point regression. The system can identify nine subjects out of ten subjects and the correct classification rate is $90 \%$. The missed subject is a healthy one. The reason is that its patterns are very similar to that of a narcoleptic subject. 
TABLE IV

PARAMETERS OF ART2 SERIES

\begin{tabular}{|c|c|c|c|c|}
\hline \multirow{2}{*}{$\begin{array}{l}\text { No. of } \\
\text { points }\end{array}$} & \multicolumn{4}{|c|}{10} \\
\hline & S1 & S2 & S3 & S4 \\
\hline $\mathrm{a}$ & 10 & 15 & 10 & 5 \\
\hline $\mathrm{b}$ & 10 & 15 & 10 & 10 \\
\hline $\mathrm{c}$ & 0.08 & 0.08 & 0.1 & 0.1 \\
\hline $\mathrm{d}$ & 0.9 & 0.9 & 0.9 & 0.9 \\
\hline$\rho$ & 0.945 & 0.94 & 0.925 & - \\
\hline $\mathrm{e}$ & 0.001 & 0.0001 & 0.00001 & 0.00001 \\
\hline \multirow{2}{*}{$\begin{array}{l}\text { No. of } \\
\text { points }\end{array}$} & \multicolumn{4}{|c|}{20} \\
\hline & S1 & S2 & S3 & S4 \\
\hline $\mathrm{a}$ & 10 & 1 & 15 & 10 \\
\hline $\mathrm{b}$ & 10 & 12 & 15 & 1 \\
\hline $\mathrm{c}$ & 0.06 & 0.07 & 0.08 & 0.1 \\
\hline $\mathrm{d}$ & 0.9 & 0.9 & 0.9 & 0.9 \\
\hline$\rho$ & 0.96 & 0.945 & 0.994 & - \\
\hline $\mathrm{e}$ & 0.001 & 0.0001 & 0.00001 & 0.00001 \\
\hline \multirow{2}{*}{$\begin{array}{l}\text { No. of } \\
\text { points }\end{array}$} & \multicolumn{4}{|c|}{25} \\
\hline & S1 & S2 & S3 & S4 \\
\hline $\mathrm{a}$ & 10 & 5 & 5 & 2 \\
\hline $\mathrm{b}$ & 10 & 15 & 10 & 10 \\
\hline $\mathrm{c}$ & 0.06 & 0.08 & 0.1 & 0.1 \\
\hline $\mathrm{d}$ & 0.9 & 0.9 & 0.9 & 0.9 \\
\hline$\rho$ & 0.9 & 0.95 & 0.945 & - \\
\hline $\mathrm{e}$ & 0.001 & 0.0001 & 0.00001 & 0.00001 \\
\hline \multirow{2}{*}{$\begin{array}{l}\text { No. of } \\
\text { points }\end{array}$} & \multicolumn{4}{|c|}{30} \\
\hline & S1 & $\mathrm{S} 2$ & S3 & S4 \\
\hline $\mathrm{a}$ & 4 & 2 & 14 & 5 \\
\hline $\mathrm{b}$ & 10 & 7 & 10 & 10 \\
\hline $\mathrm{c}$ & 0.08 & 0.08 & 0.1 & 0.1 \\
\hline d & 0.9 & 0.9 & 0.9 & 0.9 \\
\hline$\rho$ & 0.935 & 0.965 & 0.97 & - \\
\hline $\mathrm{e}$ & 0.001 & 0.0001 & 0.00001 & 0.00001 \\
\hline \multirow{2}{*}{$\begin{array}{l}\text { No. of } \\
\text { points }\end{array}$} & \multicolumn{4}{|c|}{40} \\
\hline & S1 & $\mathrm{S} 2$ & S3 & S4 \\
\hline $\mathrm{a}$ & 4 & 2 & 15 & 5 \\
\hline $\mathrm{b}$ & 10 & 7 & 10 & 10 \\
\hline $\mathrm{c}$ & 0.08 & 0.08 & 0.1 & 0.1 \\
\hline $\mathrm{d}$ & 0.9 & 0.9 & 0.9 & 0.9 \\
\hline$\rho$ & 0.985 & 0.967 & 0.97 & - \\
\hline $\mathrm{e}$ & 0.001 & 0.0001 & 0.00001 & 0.00001 \\
\hline
\end{tabular}

Each point represents $2 \mathrm{~s}$. ART2 parameters do not have any units.

\section{CONCLUding REMARKS}

We have shown that ART2 NN series can successfully classify subjects with/without OSA and subjects with/without narcolepsy based on the idea that patients with sleep disorder have different level of wakefulness from healthy people. In order to be consistent with the method by Yoss et al. [46], Keegan and Merritt standardized the conditions for measuring pupil oscillation and miosis by the pupillometric alertness level test, during which the person that is instructed to relax but try to stay awake without doing anything special is seated quietly in a dark room with eyes open and fixated on a small red spot of light. Simultaneously, the pupil diameters are measured continuously for up to $15 \mathrm{~min}$. In the data used in this paper, EEG, EMG, and eye blinks were also measured at the same time. Some studies of sleepiness in subjects with OSA have found that participants were unaware of the extent of their sleepiness under the same circumstance [11], [15] while studies on attention find that patients with narcolepsy have slower reaction times than controls, even in a relatively simple task, such as four-choice reaction time tasks [16], [41]. It is also frequently reported that perfor-

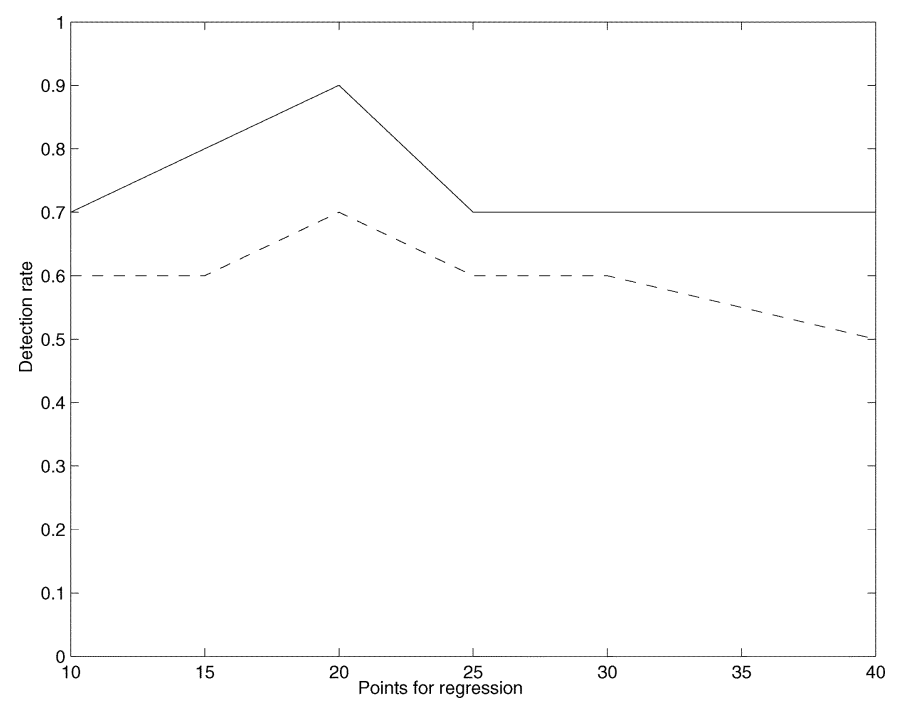

Fig. 11. Performance of one traditional ART2 NN and our system. Solid line represents performance of our system; dashed line represents performance of one traditional ART2.

mance in patients with narcolepsy is more variable than performance in controls [39], [41]. Thus, patterns of theta energy ratio in EEG can reflect the difference between sleep disorder patients and healthy people since there is good evidence that rising theta EEG activity is a sign of increasing sleepiness [21].

Although this method is for the classification of sleep disorders of OSA or narcolepsy, there is reason to believe that other sleep disorders like mixed apnea can be characterized by our system based on ART2 as well. The evidence is that the level of wakefulness for people with sleep disorders is less than that for healthy people during daytime since wakefulness represents the mental activity. EEG signals can indicate the mental activity. EEG signals in association with pupil size data provide better information for identifying sleepiness associated with sleep disorders. Eye closures and other artifacts in recorded pupil diameter data normally exist and have a severe effect on further analysis, so it is necessary to eliminate them completely from the original data.

O'Neill et al. [34] classified narcoleptic subjects and controls based on unmodeled random noise in the pupil light response. They estimated pupil noise by statistical procedures which yielded unbiased noise measures in the form of 6-D Gaussian vectors. Each subject is associated with a Gaussian vector which is optimally projected onto a scalar axis so as to maximize the mean square distance between the narcoleptic and control samples. Then, a Kullback-Leibler discrimination function was estimated and evaluated for each projection as a means of discriminating narcoleptics from controls. Since pupil noise [26] may be affected by measurement equipment and the environment, the stability of results needs to be investigated further, although good results [34] have been reported for small samples. As we know, the method of [34] strongly depends on measurement and may not be robust. ART2 NNs are robust methods to do pattern recognition. To the best of our knowledge, no similar method has been used to detect sleep disorders including OSA and narcolepsy based on EEG and pupil size. 
A series of ART2 NNs are necessary to get a precise classification result in order to eliminate effects of input ordering and to group more similar subjects together. Our experiments show that a hierarchical system of ART2 NNs could improve the accuracy of classification over that of a single ART2 network, achieving $91 \%$ between subjects with OSA and controls and achieving $90 \%$ between narcoleptic subjects and controls. The reason is that our system has more flexibility to adapt to input patterns.

\section{ACKNOWLEDGMENT}

The authors would like to thank J. A. Piscopo, who collected the data used in this research.

\section{REFERENCES}

[1] D. Aeschbach and A. A. Borbely, "All-night dynamics of the human sleep EEG,” J. Sleep Res., vol. 2, pp. 70-81, 1993.

[2] "The International Classification of Sleep Disorders: Diagnostic and Coding Manual (Revised)," American Sleep Disorders Association, Diagnostic Classification Steering Committee, 1997, Rochester, MN.

[3] R. Broughton, "Neurology and sleep research," Can. Psych. Assoc. J., vol. 16, pp. 283-293, 1971.

[4] R. Broughton, H. Yan, and B. Boucher, "Effects of one night of sleep deprivation on quantified EEG measures," J. Sleep Res., vol. 7, no. Supplement 2, p. 32, 1998.

[5] G. A. Carpenter and S. Grossberg, "ART 2: Self-organization of stable category recognition codes for analog input patterns," Appl. Opt., vol. 26, no. 23, pp. 4919-4930, 1987.

[6] M. A. Carskadon and W. C. Dement, "Multiple sleep latency test during the constant routine," Sleep, vol. 15, pp. 396-399, 1992.

[7] M. A. Carskadon and W. C. Dement, "Sleep tendency: An objective measure of sleep loss," Sleep Res., vol. 6, p. 200, 1977.

[8] M. A. Carskadon, W. C. Dement, M. M. Mitler, T. Roth, P. R. Westbrook, and S. Keenan, "Guidelines for the multiple sleep latency test (MSLT): A standard measure of sleepiness," Sleep, vol. 9, pp. 519-524, 1986.

[9] S. Chatterjee and A. S. Hadi, "Influential observations, high leverage points, and outliers in linear regression," Statist. Sci., vol. 1, pp. 379-393, 1986.

[10] M. Chen, A. A. Ghorbani, and V. C. Bhavsar, "Incremental communication for adaptive resonance theory networks," IEEE Trans. Neural Netw., vol. 16, no. 1, pp. 132-144, Jan. 2005.

[11] R. D. Cherivin and C. Guilleminault, "Obstructive sleep apnea and related disorders," Neurol. Clinics, vol. 14, pp. 583-609, 1996.

[12] M. P. Davenport and A. H. Titus, "Multilevel category structure in the ART-2 network," IEEE Trans. Neural Netw., vol. 15, no. 1, pp. 145-158, Jan. 2004.

[13] K. A. Davis and K. R. Luchten, "Respiratory impedance spectral estimation for digitally created random noise," Ann. Biomed. Eng., vol. 19 , no. 2, pp. 179-195, 1991.

[14] A. B. DuBios, A. W. Brody, D. H. Lewis, and B. F. Brugess, "Oscillation mechanics of lungs and chest in man," J. Appl. Physiol., vol. 8, pp. $587-594,1956$.

[15] H. M. Engleman and W. S. Douglas, "Under reporting of sleepiness and driving impairment in patients with sleep apnea/hypopnea syndrome," J. Sleep Res., vol. 6, pp. 272-275, 1997.

[16] R. Godbout and J. Montplaisir, "All day performance variations in normal and narcoleptic subjects," Sleep, vol. 9, pp. 200-204, 1986.

[17] S. Grossberg, "Adaptive pattern classification and universal recoding, II: Feedback, expectation, olfaction, and illusions," Biol. Cybern., vol. 23, pp. 187-202, 1976.

[18] C. Guilleminault, "Narcolepsy," in Sleep Disorders Medicine: Basic Science, Technical Considerations and Clinical Aspects, S. Chokrovery, Ed. Boston, MA: Butterworth-Heinemann, 1994, pp. 241-254.

[19] P. Y. Gunery, H. Roux-Buisson, S. Meignen, F. L. Comyn, M. Dematteis, B. Wuyam, J. L. Pepin, and P. Levy, "An adaptive detector of genioglossus EMG reflex using Berkner transform for time latency measurement in OSA pathophysiological studies," IEEE Trans. Biomed. Eng., vol. 52, no. 8, pp. 1382-1389, Aug. 2005.

[20] J. Hasan, "Past and future of computer-assisted sleep analysis and drowsiness assessment," J. Clin. Neurophysiol., vol. 13, pp. 295-313, 1996.
[21] J. A. Horne and L. A. Reyner, "Driver sleepiness," J. Sleep Res., vol. 4, pp. 23-29, 1995.

[22] A. Kales, C. R. Soldators, E. O. Bixler, A. Caldwell, R. Cadieux, J. Verrechio, and J. D. Kales, "Narcolepsy-cataplexy: II. Psychosocial consequences and associated psychopathology," Arch. Neurology, vol. 39, pp. 169-171, 1982.

[23] A. Kales, A. Vela-Bueno, and J. Kales, "Sleep disorders: Sleep apnea and narcolepsy," Ann. Intern. Med., vol. 106, pp. 434-443, 1987.

[24] A. Keegan and S. L. Merritt, "A discrete-time model to test links between EEG power and pupil diameter measured by infrared cameras," in Proc. Opt. Eng. Midwest, Aug. 1995, vol. 2622, pp. 728-735.

[25] C. R. Kollarits, F. J. Kollarits, and W. H. Schuette, "The pupil dark response in normal volunteers," Current Eye Res., vol. 2, no. 4, pp. $255-259,1982$

[26] S. Lloyd, S. Merritt, and H. Schnyders, "Does pupil noise discriminate between control and sleep-disordered subjects?," Sleep, vol. 26, pp. A273-A274, 2003.

[27] S. Lloyd, F. Meyer, B. Vern, C. Olopade, S. Merritt, and J. Shaver, "Pupillary unrest in sleep disordered versus non-sleepy healthy subjects," Sleep, vol. 28, p. A323, Jun. 2005.

[28] J. W. McLaren, W. H. Fjerstad, A. B. Ness, M. D. Graham, and R. F. Brubaker, "New video pupillometer," Opt. Eng., vol. 34, no. 3, pp. 676-682, 1995.

[29] W. B. Mendelson, Human Sleep: Research and Clinical Care. New York: Plenum, 1987, ch. 7, pp. 221-231.

[30] S. L. Merritt, A. Keegan, and P. Mercer, "Artifact management in pupillometry," Nursing Res., vol. 43, pp. 56-59, 1994.

[31] J. R. Millán, J. Mouriño, M. Franzé, F. Cincotti, M. Varsta, J. Heikkonen, and F. Babiloni, "A local neural classifier for the recognition of EEG patterns associated to mental tasks," IEEE Trans. Neural Netw., vol. 13, no. 3, pp. 678-686, May 2002.

[32] National Center on Sleep Disorders Research and Office of Prevention, Education, and Control, "Working group report on problem sleepiness," Washington, DC, 1997.

[33] National Commission on Sleep Disorders Research, "Wake-up America: A national sleep alert," Washington, DC, 1993.

[34] W. D. O'Neill, A. M. Oroujeh, and S. L. Merritt, "Pupil noise is a discriminator between narcoleptics and controls," IEEE Trans. Biomed. Eng., vol. 45, no. 3, pp. 314-322, Mar. 1998.

[35] M. Partinen and C. Hublin, "Epidemiology of sleep disorders," in Principles and Practices of Sleep Medicine, M. H. Kryger, T. Roth, and W. C. Dement, Eds., 3rd ed. Philadelphia, PA: W. B. Saunders, 2000, pp. 558-579.

[36] S. Redline, K. Kump, P. V. Tishler, I. Borwner, and V. Ferrett, "Gender differences in sleep disordered breathing in a community-based sample," Amer. J. Respiratory Critical Care Med., vol. 149 , pp. 722-726, 1994.

[37] S. Redline, P. V. Tishler, M. Schluchter, J. Aylor, K. Clark, and G. Graham, "Risk factors for sleep-disordered breathing in children: Associations with obesity, race, and respiratory problems," Amer. J. Respiratory Critical Care Med., vol. 159, no. 5, pp. 1527-1532, 1999.

[38] P. R. Roth, "Effective measurements using digital signal analysis," IEEE Spectrum, no. 1, pp. 62-70, Jan. 1971.

[39] H. Schulz and J. Wilde-Frenz, "The disturbance of cognitive processes in narcolepsy," Sleep Res., vol. 4, pp. 10-14, 1995.

[40] Y. Suzuki, "Self-organizing QRS-wave recognition in ECG using neural networks," IEEE Trans. Neural Netw., vol. 6, no. 6, pp. 1469-1477, Nov. 1995.

[41] V. Valley and R. Broughton, "Daytime performance deficits and physiological vigilance in untreated patients with narcolepsy-cataplexy compared to controls," Revue D'Electroencephalographie et de Neuophysiologie Clinique, vol. 11, pp. 133-139, 1981.

[42] P. Varady, T. Micsik, S. Benedek, and Z. Benyo, "A novel method for the detection of apnea and hypopnea events in respiration signals," IEEE Trans. Biomed. Eng., vol. 49, no. 9, pp. 936-942, Sep. 2002.

[43] A. N. Vgontzas and A. Kales, "Sleep and its disorders," Annu. Rev. Med., vol. 50, no. 1, pp. 387-400, 1999.

[44] N. Vlajic and H. C. Card, "Vector quantization of images using modified adaptive resonance algorithm for hierarchical clustering," IEEE Trans. Neural Netw., vol. 12, no. 5, pp. 1147-1162, Sep. 2001.

[45] F. C. Yen, K. Behbehani, E. A. Lucas, J. R. Burk, and J. P. Axe, "A noninvasive technique for detecting obstructive and central sleep apnea," IEEE Trans. Biomed. Eng., vol. 44, no. 12, pp. 1262-1268, Dec. 1997.

[46] R. E. Yoss, N. J. Moyer, and R. W. Hollenhorst, "Pupil size and spontaneous pupillary waves associated with alertness, drowsiness and sleep," Neurology, vol. 20, pp. 545-554, 1970.

[47] T. Young, M. Palta, J. Dempsey, J. Skatrud, S. Weber, and S. Badur, "The occurrence of sleep-disordered breathing among middle-aged adults," New England J. Med., vol. 328, pp. 1230-1235, 1993

[48] F. Zorick, T. Roehrs, R. Wittig, J. Lamphere, J. Sicklesteel, and T. Roth, "Sleep-wake abnormalities in narcolepsy," Sleep, vol. 9, pp. 189-193, 1986 


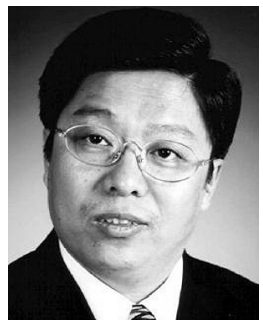

Derong Liu (S'91-M'94-SM'96-F'05) received the $\mathrm{Ph} . \mathrm{D}$. degree in electrical engineering from the University of Notre Dame, Notre Dame, IN, in 1994.

From 1993 to 1995, he was a Staff Fellow with General Motors Research and Development Center, Warren, MI. From 1995 to 1999, he was an Assistant Professor in the Department of Electrical and Computer Engineering, Stevens Institute of Technology, Hoboken, NJ. He joined the University of Illinois at Chicago in 1999, where he is now a Full Professor of electrical and computer engineering and of computer science. Since 2005, he has been Director of Graduate Studies in the Department of Electrical and Computer Engineering, University of Illinois at Chicago. He is coauthor (with A. N. Michel) of Dynamical Systems with Saturation Nonlinearities: Analysis and Design (New York: Springer-Verlag, 1994), (with A. N. Michel) Qualitative Analysis and Synthesis of Recurrent Neural Networks (New York: Marcel Dekker, 2002), and (with H. G. Zhang) Fuzzy Modeling and Fuzzy Control (Boston, MA: Birkhauser, 2006). He is coeditor (with P. J. Antsaklis) of Stability and Control of Dynamical Systems with Applications (Boston, MA: Birkhauser, 2003), (with F. Y. Wang) Advances in Computational Intelligence: Theory and Applications (Singapore: World Scientific, 2006), and (with S. M. Fei, Z.-G. Hou, H. G. Zhang, and C. Y. Sun) Advances in Neural Networks ISNN2007 (Berlin, Germany: Springer-Verlag, 2007).

Dr. Liu is an Associate Editor of Automatica. He was the General Chair for the 2007 International Symposium on Neural Networks (Nanjing, China). He was a member of the Conference Editorial Board of the IEEE Control Systems Society (1995-2000) and an Associate Editor of the IEEE TRANSACTIONS ON CIRCUITS AND SYSTEMS-PART I: FUNDAMENTAL THEORY AND APPLICATIONS (1997-1999), the IEEE TRANSACTIONS ON SigNAL PROCESSING (2001-2003), and the IEEE TRANSACtions ON NeURAL Networks (2004-2006). Since 2004, he has been the Editor of the IEEE Computational Intelligence Society's ELECTRONIC LETTER. Since 2006, he has been the Letters Editor of the IEEE TRANSACTIONS ON NEURAL NETWORKS and an Associate Editor of the IEEE Computational Intelligence Magazine. He is the General Chair for the 2008 IEEE International Conference on Networking, Sensing and Control. $\mathrm{He}$ is the Program Chair for the 2008 International Joint Conference on Neural Networks; the 2007 IEEE International Symposium on Approximate Dynamic Programming and Reinforcement Learning; the 21st IEEE International Symposium on Intelligent Control (2006); and the 2006 International Conference on Networking, Sensing and Control. He has served and is serving as a member of the organizing committee and the program committee of several other international conferences. He is an elected AdCom member of the IEEE Computational Intelligence Society (2006-2008), Chair of the Chicago Chapter of the IEEE Computational Intelligence Society, and Past Chair of the
Technical Committee on Neural Systems and Applications of the IEEE Circuits and Systems Society. He received the Michael J. Birck Fellowship from the University of Notre Dame (1990), the Harvey N. Davis Distinguished Teaching Award from Stevens Institute of Technology (1997), the Faculty Early Career Development (CAREER) award from the National Science Foundation (1999), and the University Scholar Award from University of Illinois (2006-2009). He is a member of Eta Kappa Nu.

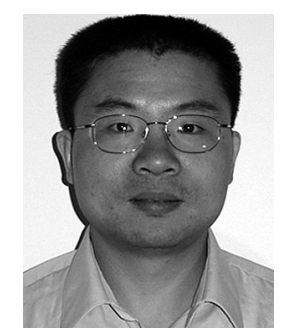

Zhonyu Pang (S'06) received the M.S. degree in electrical engineering from Oklahoma State University, Stillwater, in 2003. Currently, he is working towards the Ph.D. degree at the Department of Electrical and Computer Engineering, University of Illinois at Chicago, Chicago.

His research interests include biomedical signal processing, epileptic seizure prediction, artificial intelligence, pattern recognition, and bioinformatics.

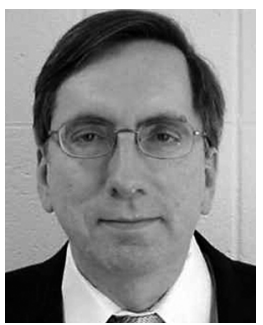

Stephen R. Lloyd received the B.S. degree in physics from Illinois Institute of Technology, Chicago, in 1969 and the Ph.D. degree in psychology from the University of Illinois at Chicago, Chicago, in 1986.

Currently, he is an Adjunct Assistant Professor at the College of Nursing, University of Illinois at Chicago, Chicago. From 1969 to 1973, he worked as a Quality Assurance Engineer in the Aerospace Electronics Division of the General Electric Company. During and following graduate school, he performed research relating to sleep disorders and quality of life issues in cancer and other medical disorders. He has authored and coauthored more than 20 journal publications and book chapters, as well as presentations at professional conferences. He has also worked on numerous research projects as an independent consultant on experimental design and statistical analysis, as well as custom research equipment hardware design and software programming. He holds four U.S. patents on medical electronic devices. 